\title{
Sweat as a Source of Next-Generation Digital Biomarkers
}

\author{
Noé Brasiera, bens Eckstein ${ }^{a, c}$ \\ ${ }^{a} \mathrm{CMIO}$ Research Group, University Hospital Basel, Basel, Switzerland; \\ ${ }^{b}$ Department of Internal Medicine, Kantonsspital Obwalden, Sarnen, Switzerland; \\ 'Department of Internal Medicine, University Hospital Basel, Basel, Switzerland
}

\section{Keywords}

Sweat · Digital biomarkers · Wearables $\cdot$ iSudorology

\begin{abstract}
Sweat has been associated with health and disease ever since it was linked to high body temperature and exercise. It contains a broad range of electrolytes, proteins, and lipids, and therefore hosts a broad panel of potential noninvasive biomarkers. The development of novel smartphone-based biosensors will enable a more sophisticated, patient-driven sweat analysis. This will provide a broad range of novel digital biomarkers. Digital biomarkers are of increasing interest because they deliver various relevant longitudinal health data. To date, investigations on digital biomarkers have focused on creating objective measurements of function. Sweat analysis using smartphone-based biosensors has the potential to provide initial noninvasive metabolic feedback and therefore represents a promising complement and a source for next-generation digital biomarkers. From this viewpoint, we discuss stateof-the-art sweat research, focusing on the clinical implementation of sweat in medicine. Sweat provides biomarkers that represent direct metabolic feedback and is therefore expected to be the next generation of digital biomarkers. With regard to its broad application in various fields of medicine, we see a clear need to evolve the internet-enabled field of sweat expertise: iSudorology.

\section{Introduction}

Sweat is a noninvasively collectable biofluid that hosts a broad range of biomarkers. Sweat not only helps the body to cool down but also provides information about a person's metabolic state. However, blood analysis is established and highly standardized, and blood is the standard biofluid when it comes to physiologic feedback analysis. The investigation of 
sweat has been fascinating researchers for many years, but the high demands on laboratory infrastructure have thus far prevented the clinical implementation of sweat as a diagnostic biofluid. Nevertheless, interest in the analysis and investigation of sweat components has been increasing throughout the past century, leading to the current importance of sweat as a potential diagnostic biofluid.

In 1910, Embden [1] demonstrated the existence of the amino acid serine in human sweat. Since then, an increasing number of investigations aiming to determine the composition of human sweat has been undertaken. In the early 20th century, Silvers et al. [2] detected glucose in sweat and blood. McSwiney [3] was able to isolate ammonia, glucose, and chloride, for example, and was one of the first to compare sweat components between healthy people and rheumatoid patients in 1934. In 1969, Johnson and Shuster [4] described reduced hydrosis and electrolyte excretion in psoriasis-affected skin areas of psoriasis patients and opened up the field of sweat analysis in dermatology. Only a few years later, Förström et al. [5] detected prostaglandins in sweat, thereby further identifying an important biomarker for inflammation. In 1970, Peter et al. [6] proved the appearance of C12-C22 fatty acids in sweat and thus added the class of lipophilic compounds to the repertoire of available biomarkers in sweat. Prompt et al. [7] investigated the sweat of patients with renal failure and were able to show a significant increase in magnesium, calcium, and phosphate, thus indicating diseasespecific changes in sweat ion concentrations. Despite the presence of different disease-indicating metabolites in sweat, in 1992 Wormser et al. [8] proved the absence of HIV in human eccrine sweat. This sweat investigation had a strong impact on the safety and prevention of HIV transmission.

Proteomic and metabolomic technologies now enable sweat analysis with unprecedented sensitivity and numbers of detected metabolites at the same time. Yu et al. [9] detected more than 800 unique proteins and 32,000 endogenous peptides in sweat and opened up an exciting field of potential novel, noninvasive biomarkers. Despite these promising results, there are challenges to overcome, such as the difficulty in sampling and laborious analytics. These issues are still preventing sweat analytical approaches from a breakthrough in clinical diagnostics.

Emerging smartphone-based biosensors allow for the easy detection of a variety of physiologic metrics [10], including sweat markers [11], and they are already broadly available. As sweat sampling and analysis have been major obstacles for broad clinical investigation and application, smartphone-based sensors have the potential to be the missing link that will open up a new field of internet-enabled sweat analysis in research and clinics.

In this article, we provide an overview of state-of-the-art sweat sampling and analysis as well as detail the upcoming smartphone-based biosensors for sweat analysis. Further, we introduce the latest advances in clinical sweat research and diagnostics. At the end of this overview, we point out why we see great potential in sweat biomarkers as next-generation digital biomarkers in health care and the demand to further accelerate and explore new fields of sweat analysis.

\section{Sweat Sampling}

As sweat appears on the skin surface, noninvasive collection is possible, which provides a clear advantage over state-of-the-art invasive blood sampling. Sweat is collected passively using sweat patches or is actively induced by medical device grade sweat-sampling devices. Active induction is carried out by the topical application of a sweat gland-stimulating substance as well as local current [12]. Despite being noninvasive, current standard sweat sampling remains a challenge, as sample volumes are mostly small and metabolite concen- 
tration low. Exercise to induce acute sweating and collect larger amounts of sweat is a potential approach to achieve larger sample volumes but is mostly restricted to healthy subjects.

Different skin areas show various proportions of different sweat glands (eccrine, apocrine, and holocrine) that own their proper secretion mechanisms. Therefore, each type of sweat gland excretes a different quantity and quality of biomarkers. As a consequence, the detection of specific metabolites and drugs depends greatly on the body location where the sample is collected [13]. Sweat sampling is mostly conducted on the volar lower arm along the eccrine sweat glands of the skin. Those sampling sites are well accessible and contain a homogenous distribution of eccrine sweat glands. This enables a standardized collection process.

\section{Sweat Analysis}

Currently, the 2 most common analytic methods for sweat analysis in the laboratory are mass spectrometry [9], which detects a broad range of metabolites at the same time, and the much cheaper enzyme-linked immunosorbent assay (ELISA) with a clearly defined target molecule [14]. Despite the advantage of mass spectrometry to be able to detect a wide range of biomarkers at the same time, from a clinical point of view it remains laboratory dependent, costly, and time consuming. In general, sweat samples need to be stabilized as well as stored at $-80^{\circ} \mathrm{C} /-112^{\circ} \mathrm{F}$ to prevent molecular degradation.

Smartphone-based biosensors are still in development, as receptor cleansing and stability over time as well as the detection of molecules present at low concentrations are major challenges [15]. From a developer perspective, it is easier to develop a disposable patch for spot measurements because cleansing and maintenance challenges are omitted before the investigation in favor of a sustainable biosensor that remains on the skin for a longer period. Further, a qualitative measurement aiming to determine either the presence or the absence of a digital biomarker will be a more easily achievable task in the beginning versus continuous quantitative measurements. The assessment of exact concentration changes over time is highly desirable and clinically important but comes with much higher demands on biosensor sustainability and validity. Progress has been made in this highly active research field, and a novel solution for passive sweat analysis in spot ELISA-based receptors using a multiplex capture-antibody microarray is under development. This solution detects a set of up to 6 proteins and analyzes sweat compounds independent of the laboratory [16]. Alternatively, a wearable sweat sensor based on iontophoresis is being developed. This device enables analysis of actively induced sweat [17]. The 2 aforementioned biosensors are linked to smartphones that display and transfer sensor data instantly. They therefore do not need any personal contact with a health care worker $[18,19]$. However, the clinical implementation and in situ application of sweat sensors in health care have not been achieved yet. In 2016, Liu et al. [20] successfully conducted not only artificial sweat trials but also an in situ, realtime, proof-of-concept human sweat analysis. They measured sweat conductivity in 4 participants riding a stationary exercise bike in a laboratory and wearing an on-skin biosensor. An HTMC PM23300 Windows phone served as the monitoring terminal. Nevertheless, there are increasing numbers of upcoming sensors along with novel approaches to automatically and artificially test those devices [21] and to fabricate cost-effective sensors on a large scale [22]. These are all significant requirements that pave the way for a successful clinical implementation of wearable sweat sensors.

The electrochemical properties of molecules (charge, hydrophilic, and lipophilic qualities) influence the secretion of a metabolite into sweat. Those mechanisms have already been discussed in detail by Sonner et al. [23]. It is important to mention that the excretion process 
Brasier and Eckstein: Digital Biomarkers in Sweat

of sweat biomarkers is dependent on active and/or passive channels in the case of hydrophilic qualities or passive diffusion through the skin barrier in case of lipophilic qualities [24]. Those mechanisms need to be taken into account before investigating sweat compounds as biomarkers.

Despite the aforementioned limitations of current standard sweat analysis, mass spectrometry in particular enables a hypothesis-driven investigation to further reveal the potential of sweat as a diagnostic biofluid.

\section{Clinical Sweat Research}

As mentioned in the introduction, different approaches have been used to investigate sweat composition in health and disease. Sweat hosts different disease-specific markers and drug metabolites. Recently, an increasing number of investigations has been undertaken to reveal the potential of sweat as a source of biomarkers, which will be discussed in this section. In the following examples, we will provide an overview of the current research and potential impact of sweat as a biofluid in clinical diagnostics.

\section{Infectious Diseases}

Tuberculosis is the ninth leading cause of death worldwide and the deadliest single infectious agent [25]. Diagnosis of tuberculosis requires an extended laboratory infrastructure and remains challenging, because it is sputum dependent and not every patient is able to produce sputum. Most patients suffering from tuberculosis reside in low-income countries with restricted, distantly located health care infrastructure [25]. Active tuberculosis is associated with night sweats, which suggests an association between sweat changes and disease. Indeed, Adewole et al. [26] investigated the sweat composition of patients with active tuberculosis and detected 26 disease-specific sweat proteins. These results need to be confirmed in different trials with established robust test criteria for the detection of active tuberculosis. This sweat-based test to screen active tuberculosis would enable the classification of subjects at high risk and determine the need for extended diagnostics in remote settings without health care access. As patients sometimes need to travel for hours to the next hospital for diagnosis, this screening device could support a reasonable risk assignment. It could be further used as an additional exclusion tool, as the diagnosis of active tuberculosis can take weeks depending on the patient's clinic.

\section{Immunology}

The human skin hosts different cytokine receptors and plays an active role in the immune response. Therefore, the presence of a broad range of cytokines in sweat samples is not unexpected. Katchman et al. [27] detected immunoglobulin A (IgA), IgD, IgG, and IgM, which suggests the possibility to profile antibodies as well as innate immune reactions in sweat. Earlier, Dai et al. [28] described immunoglobulins in sweat-activating epidermal keratinocytes if skin is affected. Successful correlation between serum and sweat concentrations of different cytokines in healthy women by Marques-Deak et al. [29] indicates the potential of sweat analysis to further serve as a noninvasive, diagnostic method to monitor inflammatory response. Psoriasis is a skin condition in young and older patients that leads to highly itchy inflammatory exanthema, arthritis, and other effects with a change in skin immunologic response by upregulating proinflammatory cytokines among others [30]. A patient-driven noninvasive sweat analysis to detect, for example, changes in proinflammatory cytokines in sweat would allow a personalized anti-inflammatory treatment regimen, which can be essential in certain comorbidities, such as heart or kidney failure. 
Brasier and Eckstein: Digital Biomarkers in Sweat

\section{Neurology}

Parkinson disease has shown an increasing prevalence and is responsible for the highest number of deaths and disability among neurodegenerative diseases. General screening for Parkinson disease is based on clinical symptoms, therefore leading to a delay in the initial diagnosis. A noninvasive, easily applicable screening method to detect the disease at an early stage is missing. In one study, the wife of a Parkinson disease patient reported a smell of musk on her husband, who was diagnosed with Parkinson disease later on. Driven by this observation, Trivedi et al. [31] investigated the sebum of Parkinson disease patients and tested it with the help of the mentioned patient's wife. As a result, they were able to isolate 4 Parkinson disease-specific proteins. A sweat-based noninvasive screening test for Parkinson disease will provide a novel tool for detecting patients at an early stage of the disease. Even though Parkinson disease is not curable at this time, earlier disease detection will provide novel insights into initial molecular changes, reveal novel treatment targets, as well as provide a better understanding of the disease.

\section{Psychiatry}

Schizophrenia has a median incidence of about $15.2 / 100,000$ people [32]. It is a complex disease with a mix of positive symptoms (e.g., hallucinations) and negative symptoms (e.g., lack of emotional responsiveness). Despite several investigations, a specific biomarker for schizophrenia is still missing. This makes the initial diagnosis difficult, as differentiating between schizophrenia and other psychiatric diagnoses is a challenge, especially in early phase disease. Early diagnosis is crucial because an adequate first-line treatment has a significant impact on long-term outcome and disease chronification [33]. Therefore, a noninvasive biomarker indicating disease is desirable. In 1960, Smith and Sines [34] investigated a peculiar odor coming from patients suffering from schizophrenia and demonstrated that rats can differ between sweat samples from schizophrenia patients and controls. Recently, Raiszadeh et al. [35] demonstrated different protein excretion in eccrine sweat of schizophrenic patients. If results are replicated and disease-specific biomarkers further defined, early detection of the disease in prodromal patients will provide a clear indication for antipsychotic treatment. In addition, behavioral therapy (for example) can be established early to prevent behavioral risk factors, such as sleep deprivation. As schizophrenic patients have a risk of relapse mostly due to treatment noncompliance, a noninvasive sweat test can be used to detect biomarker changes and potentially serve as an indirect marker to monitor treatment adherence. Nevertheless, strict testing to investigate the reliability of these markers to differentiate from other psychiatric diseases will be essential. This is also of high importance because antipsychotic and antidepressant drugs can lead to significant side effects.

\section{Endocrinology}

Diabetes mellitus is an epidemic with a prevalence ranging from 10 to $38 \%$ depending on the region [36]. The impact of diabetes mellitus-related comorbidities on the health of the young and old is substantial. Currently, measurement of blood glucose relies on semi-invasive finger prick serum analysis. Upcoming minimally invasive glucose monitoring remains up to 14 days in a patient's subcutis. A fully noninvasive device is still missing but highly favorable to increase compliance and therapy adherence. Optimal treatment prevents diabetes mellitusrelated short- and long-term comorbidities. Silvers et al. [2] demonstrated simultaneous changes of glucose in sweat and blood and was able to track the intake of an excessive 2-pound chocolate cake in early 1928. More recently, Moyer et al. [37] confirmed the correlation between sweat and blood glucose levels. This promising approach of noninvasive, continuous glucose monitoring has the potential to enable higher monitoring resolution to adapt insulin therapy and prevent hypo- and hyperglycemia. 
Brasier and Eckstein: Digital Biomarkers in Sweat

\section{Pulmonology}

Cystic fibrosis is an inherited disease that affects multiple organ systems, predominantly the lungs and pancreas, with a median life expectancy of 40 years [38]. As a consequence, patients with cystic fibrosis suffer primarily from recurrent pulmonary infections as well as malabsorption. Initial diagnosis is obtained by sweat analysis, and disease severity can differ between patients along with different genetic appearances. Detecting altered chloride concentrations in the sweat of cystic fibrosis patients represents the only clinical standard diagnostic sweat test [39].

\section{Nephrology}

As mentioned in the introduction, there have been different investigations to detect concentration changes of specific electrolytes in the sweat of patients with chronic kidney failure [7]. Despite promising results, a clear threshold to diagnose kidney function is still missing [40]. A noninvasive test to evaluate kidney function would be highly beneficial for many clinical settings. For instance, in heart failure patients, diuretic therapy and hydration status could be monitored in clinical and outpatient settings as soon as smartphone-based biosensors are available.

\section{Oncology}

Canine scent is one of nature's most sensitive detectors of specific smells. Various trials have shown that dogs can scent bladder cancer, most likely by sensing volatile components [41]. Junqueira et al. [42] pursued a comparable approach and demonstrated the potential of dogs to differentiate between blood samples from patients with lung cancer and healthy controls. Calderon-Santiago et al. [43] detected a specific trisaccharide phosphate in human eccrine sweat specific to patients with lung cancer. With those findings, they were able to implement a metabolic marker for screening of lung cancer. This supports the indication of further investigation of sweat biomarkers to establish a reliable and noninvasive screening tool for early cancer diagnosis or recurrence detection.

\section{Pharmacology}

Various pharmacologic metabolites have been detected in sweat. Different commercially available patches that detect drugs including opioids [44], 3,4-methylenedioxymethamphetamine [45], tetrahydrocannabinol [46], and methylphenidate [47] already exist. Those patches have primarily been used to demonstrate the absence of drug abuse, mostly in a forensic setting. In a more clinical approach, Høiby et al. [13] showed the excretion of orally taken $\beta$-lactam and quinolone antibiotics in the sweat of healthy volunteers. The excreted antibiotics even showed an impact on the resistance profile of bacterial skin flora $[13,48]$. In addition to the mentioned detection of Parkinson disease-specific molecules, Tsunoda et al. [49] successfully investigated sweat as a source for noninvasive monitoring of L-dopa treatment in Parkinson disease. Despite a correlation coefficient of only 0.68 between sweat and blood concentrations, it is a very promising opportunity to optimize treatment and patient safety.

\section{Conclusion}

The use of sweat as a noninvasive, laboratory-independent, on-skin diagnostic biofluid has the potential to have a major impact on health care in the future. Investigation of sweat biomarkers has been conducted in different medical fields. Once smartphone-based biosensors are available for on-skin point-of-care sweat analysis, sweat compounds will be broadly 


\begin{tabular}{l|l|}
\hline Digit Biomark 2019;3:155-165 \\
\hline DOI: 10.1159/000504387 & $\begin{array}{l}\text { @ 2019 The Author(s). Published by S. Karger AG, Basel } \\
\text { www.karger.com/dib }\end{array}$ \\
\hline
\end{tabular}

Table 1. Overview on sweat diagnostic research

\begin{tabular}{|c|c|c|c|c|}
\hline $\begin{array}{l}\text { Medical } \\
\text { field }\end{array}$ & Disease & Sweat markers & $\begin{array}{l}\text { Blood } \\
\text { correlation }\end{array}$ & References \\
\hline $\begin{array}{l}\text { Infectious } \\
\text { diseases }\end{array}$ & Tuberculosis & $\begin{array}{l}\text { e.g., complement C1q subcomponent } \\
\text { subunit C, complement C1r } \\
\text { subcomponent, C-reactive protein }\end{array}$ & na & Adewole et al. [26] \\
\hline Immunology & Unspecific & e.g., IL- $1 \alpha$, TNF- $\alpha$, IFN- $\gamma$ & na & Katchman et al. [27] \\
\hline Neurology & $\begin{array}{l}\text { Parkinson } \\
\text { disease }\end{array}$ & $\begin{array}{l}\text { e.g., perillic aldehyde, hippuric acid, } \\
\text { eicosane }\end{array}$ & na & Trivedi et al. [31] \\
\hline Psychiatry & Schizophrenia & e.g., $\alpha_{2}$-glycoprotein, annexin 5 , arginase & Yes & Raiszadeh et al. [35] \\
\hline Endocrinology & Diabetes mellitus & Glucose & Yes & Moyer et al. [37] \\
\hline Pneumology & Cystic fibrosis & $\mathrm{Cl}$ & na & Farrell et al. [59] \\
\hline Nephrology & Kidney failure & e.g., Na, K, P & na & Prompt et al. [7] \\
\hline Oncology & Lung cancer & $\begin{array}{l}\text { e.g., trisaccharide phosphate, trihexose, } \\
\text { nonanedioic acid }\end{array}$ & na & $\begin{array}{l}\text { Calderon-Santiago et } \\
\text { al. [43] }\end{array}$ \\
\hline Pharmacology & Antibiotics & $\begin{array}{l}\text { e.g., benzylpenicillin, cefuroxime, } \\
\text { ceftriaxone }\end{array}$ & Yes & Høiby et al. [13] \\
\hline
\end{tabular}

na, not assessed in this particular study.

available as digital biomarkers. This will not only allow lab-independent monitoring but also enable the collection and sharing of personal health data. Patient-driven sweat analysis could provide the opportunity to detect metabolic changes in real-time and combine these data with context-driven patient information such as heart rate, accelerometer data, as well as respiratory data. This approach should provide deeper and longitudinal insights into physiological and pathophysiological changes. This will further enable personalized treatment regimens as well as remote patient monitoring.

Digital biomarkers are a rapidly emerging field driven by the increasing presence of smart devices, continuously improving network technology, and smart algorithms. This setting will enable health care professionals and individuals to collect longitudinal digital biomarkers from patients and healthy subjects [50,51]. So far, the most discussed digital biomarkers have been patient accelerometer data as well as photoplethysmographic data to detect, for example, tremor [52] or atrial fibrillation [53]. Both parameters are of special interest because of their employment in the consumer market for a while with robust, established biosensors; they give extended longitudinal insights into patient health and disease. Despite the transformation to an easily available smartphone-based point-of-care blood analysis in clinics [54], the current standard metabolic biomarker collection method remains invasive. The detection of noninvasive metabolic feedback by sweat analysis is a highly promising complementation to the ever-expanding range of functional biomarkers by providing direct physiological information.

Sweat has been investigated since the beginning of the 20th century but has gained increasing interest recently (Table 1 ). With the emergence of smartphone-based biosensors, diagnostic and clinical implementation of sweat analysis will become readily available in the near future. A laboratory- and clinic-independent, on-demand, and instant metabolic feedback from sweat analysis by novel smartphone-based biosensors will influence clinical and outpatient care sustainably in research, development, and clinical settings. Particularly electro- 


\begin{tabular}{l|l}
\hline Digit Biomark 2019;3:155-165 \\
\hline DOI: 10.1159/000504387 & $\begin{array}{l}\text { @ 2019 The Author(s). Published by S. Karger AG, Basel } \\
\text { www.karger.com/dib }\end{array}$ \\
\hline
\end{tabular}

Brasier and Eckstein: Digital Biomarkers in Sweat

Table 2. Overview on sweat and blood characteristics

\begin{tabular}{|c|c|c|}
\hline Characteristics & Sweat & Blood \\
\hline \multicolumn{3}{|l|}{ Sampling } \\
\hline Invasiveness & Noninvasive & Invasive \\
\hline Quality & Active vs. passive induction & Passive \\
\hline Time & $30 \min +5 \min$ (if induced) & $2 \mathrm{~s}$ \\
\hline Location & Eccrine vs. apocrine & Vein and/or artery \\
\hline Biofluid composition & $\begin{array}{l}\text { Dynamic (depending on body } \\
\text { temperature, ethnicity, and gender, } \\
\text { for example) }\end{array}$ & Basically stable \\
\hline $\begin{array}{l}\text { Concentrations, e.g., mean } \\
\text { ceftriaxone peak } \\
1 \mathrm{~h} \text { after application }\end{array}$ & $2.5 \mu \mathrm{g} / \mathrm{mL}$ (eccrine) [13] & $372 \mu \mathrm{g} / \mathrm{mL}[13]$ \\
\hline Clinical application & $\begin{array}{l}\text { Smartphone-based sweat sensors } \\
{[15-18]}\end{array}$ & $\begin{array}{l}\text { Smartphone-based } \\
\text { point-of-care analysis [54] }\end{array}$ \\
\hline
\end{tabular}

lytes, which are secreted primarily by sweat glands; smaller proteomic compounds (molecular weight $<100 \mathrm{kDa}$ ) secreted by active and passive channels [9]; and lipophilic compounds tend to be detectable in sweat compared with larger, hydrophilic molecules [23]. In summary, the ability of sweat markers to reflect a patient's metabolic state qualifies sweat as a source of next-generation digital biomarkers.

Despite the high potential, clinical use of sweat remains a point of discussion. The composition of sweat components is subject to more dynamic changes than that of blood, as evaporation, contamination on the skin surface, and other factors challenge a reliable quantification. The concentrations of sweat molecules are lower than in blood, resulting in increasing demands on biosensors, especially with respect to small sample volumes. As sweat glands are not homogenously distributed along the body surface, the specific localization of sampling needs to be chosen carefully [13]. Yet another challenge is that sweat rate and composition depend on ethnicity, gender, age, environmental factors, and physical activity (Table 2) [55, 56]. Combining sweat sensors with additional wearable biosensors could provide contextdriven patient information. Assessing, for example, a combination of a patients' accelerometer data together with heart rate and oxygen saturation will provide information on a patients' activity level. If increased, this will physiologically lead to an augmented sweat gland activity with a subsequent increase in sweat rate and vice versa. Taking into account ambient, skin, and body temperature will even enable a more differentiated contextual approach. Especially when such data could be combined with machine-learning algorithms, the resulting holistic approach could overcome many of the current limitations and provide accurate measures of standard sweat gland activity [57].

In conclusion, we see a high potential for sweat as a noninvasively collectable biofluid hosting a broad range of next-generation digital biomarkers. As demonstrated along this viewpoint, sweat markers have great potential to affect research, diagnostics, and treatment across health care fields. There are differences in sampling and metabolite concentrations between blood and sweat, which is why we see the need to evolve "iSudorology," the science of internet-enabled sweat expertise. This will accelerate investigation, development, and implementation of this promising biofluid. Research and development in the field of smartphone-based, wearable sweat biosensors has been of increasing interest $[18,19,58]$ and will be crucial for long-term implementation of sweat diagnostics in health care. 


\begin{tabular}{l|l}
\hline \multicolumn{2}{l}{ Digit Biomark 2019;3:155-165 } \\
\hline DOI: 10.1159/000504387 & $\begin{array}{l}\text { (c) 2019 The Author(s). Published by S. Karger AG, Basel } \\
\text { www.karger.com/dib }\end{array}$ \\
\hline
\end{tabular}

Brasier and Eckstein: Digital Biomarkers in Sweat

\section{Acknowledgment}

The authors would like to thank Martin Kahn and Fiorangelo De Ieso for their critical reviews.

Statement of Ethics

This study did not directly involve patients; thus ethical approval was not required.

Disclosure Statement

The authors have no conflicts of interest to declare.

Funding Sources

J. Eckstein is a staff member at the University Hospital Basel.

\section{Author Contributions}

N.B. and J.E. contributed substantially to the conception of the work as well as the critical interpretation of the literature. N.B. drafted and J.E. critically reviewed the work, and both finally approved the version to be published. Both agreed to be accountable for all aspects of the work in ensuring that questions related to the accuracy or integrity of any part of the work are appropriately investigated and resolved.

\section{References}

1 Embden G, Tachau H. Über das Vorkommen von Serin im menschlichen Schweisse. Biochem Z. 1910;28:230-6.

2 Silvers S, Forster W, Talbert GA. Simultaneous study of the constituents of the sweat, urine and blood, also gastric acidity and other manifestations resulting from sweating. Am J Physiol. 1928;84(3):577-82.

3 McSwiney BA. The composition of human perspiration (Samuel Hyde Memorial Lecture): (Section of Physical Medicine). Proc R Soc Med. 1934 May;27(7):839-48.

4 Johnson C, Shuster S. Eccrine sweating in psoriasis. Br J Dermatol. 1969 Feb;81(2):119-24.

5 Förström L, Goldyne ME, Winkelmann RK. Prostaglandin activity in human eccrine sweat. Prostaglandins. 1974 Sep; $7(6): 459-64$.

6 Peter G, Schröpl F, Feisel HG, Thürauf W. Gaschromatographische Untersuchungen von freien und gebundenen Fettsäuren im ekkrinen Schweiss. Arch Klin Exp Dermatol. 1970;238(2):154-9.

7 Prompt CA, Quinton PM, Kleeman CR. High concentration of sweat calcium, magnesium and phosphate in chronic renal failure. Nephron. 1978;20(1):4-9.

8 Wormser GP, Bittker S, Forseter G, Hewlett IK, Argani I, Joshi B, et al. Absence of infectious human immunodeficiency virus type 1 in "natural" eccrine sweat. J Infect Dis. 1992 Jan;165(1):155-8.

9 YuY, Prassas I, Muytjens CM, Diamandis EP. Proteomic and peptidomic analysis of human sweat with emphasis on proteolysis. J Proteomics. 2017 Feb;155:40-8.

10 Topol EJ, Steinhubl SR, Torkamani A. Digital medical tools and sensors. JAMA. 2015 Jan;313(4):353-4.

11 Nyein HY, Tai LC, Ngo QP, Chao M, Zhang GB, Gao W, et al. A wearable microfluidic sensing patch for dynamic sweat secretion analysis. ACS Sens. 2018 May;3(5):944-52.

12 Wescor. Macroduct sweat collection system - Model 3700 SYS. Instruction/service manual. 2004. Available from: http://www.wescor.com/translations/Translations/M2551-7A-EN.pdf.

13 Høiby N, Pers C, Johansen HK, Hansen H. Excretion of beta-lactam antibiotics in sweat-a neglected mechanism for development of antibiotic resistance? Antimicrob Agents Chemother. 2000 0ct;44(10):2855-7. 


\begin{tabular}{l|l|}
\hline Digit Biomark 2019;3:155-165 \\
\hline DOI: 10.1159/000504387 & $\begin{array}{l}\text { ○ 2019 The Author(s). Published by S. Karger AG, Basel } \\
\text { www.karger.com/dib }\end{array}$ \\
\hline
\end{tabular}

Brasier and Eckstein: Digital Biomarkers in Sweat

14 Delgado-Povedano MM, Calderón-Santiago M, Luque de Castro MD, Priego-Capote F. Metabolomics analysis of human sweat collected after moderate exercise. Talanta. 2018 Jan;177:47-65.

15 Munje RD, Muthukumar S, Jagannath B, Prasad S. A new paradigm in sweat based wearable diagnostics biosensors using room temperature ionic liquids (RTILs). Sci Rep. 2017 May;7(1):1950.

16 Orro K, Smirnova O, Arshavskaja J, Salk K, Meikas A, Pihelgas S, et al. Development of TAP, a non-invasive test for qualitative and quantitative measurements of biomarkers from the skin surface. Biomark Res. 2014 Nov; 2(1):20.

17 Emaminejad S, Gao W, Wu E, Davies ZA, Yin Yin Nyein H, Challa S, et al. Autonomous sweat extraction and analysis applied to cystic fibrosis and glucose monitoring using a fully integrated wearable platform. Proc Natl Acad Sci USA. 2017 May;114(18):4625-30.

18 Currano LJ, Sage FC, Hagedon M, Hamilton L, Patrone J, Gerasopoulos K. Wearable sensor system for detection of lactate in sweat. Sci Rep. 2018 Oct;8(1):15890.

19 Gao W, Emaminejad S, Nyein HY, Challa S, Chen K, Peck A, et al. Fully integrated wearable sensor arrays for multiplexed in situ perspiration analysis. Nature. 2016 Jan;529(7587):509-14.

20 Liu G, Ho C, Slappey N, Zhou Z, Snelgrove SE, Brown M, et al. A wearable conductivity sensor for wireless realtime sweat monitoring. Sens Actuators B Chem. 2016;227:35-42.

21 Brueck A, Bates K, Wood T, House W, Martinez Z, Peters S, et al. An artificial sweating system for sweat sensor testing applications. Electronics (Basel). 2019;8(6):606.

22 Nyein HY, Bariya M, Kivimäki L, Uusitalo S, Liaw TS, Jansson E, et al. Regional and correlative sweat analysis using high-throughput microfluidic sensing patches toward decoding sweat. Sci Adv.2019 Aug;5(8):eaaw9906.

23 Sonner Z, Wilder E, Heikenfeld J, Kasting G, Beyette F, Swaile D, et al. The microfluidics of the eccrine sweat gland, including biomarker partitioning, transport, and biosensing implications. Biomicrofluidics. 2015 May; 9(3):031301.

24 Sato K, Kang WH, Saga K, Sato KT. Biology of sweat glands and their disorders. I. Normal sweat gland function. J Am Acad Dermatol. 1989 Apr;20(4):537-63.

25 World Health Organization. Global tuberculosis report 2018 [Internet]. Geneva: World Health Organization; 2018.

26 Adewole 00, Erhabor GE, Adewole TO, Ojo AO, Oshokoya H, Wolfe LM, et al. Proteomic profiling of eccrine sweat reveals its potential as a diagnostic biofluid for active tuberculosis. Proteomics Clin Appl. 2016 May; 10(5):547-53.

27 Katchman BA, Zhu M, Blain Christen J, Anderson KS. Eccrine sweat as a biofluid for profiling immune biomarkers. Proteomics Clin Appl. 2018 Nov;12(6):e1800010.

28 Dai X, Okazaki H, Hanakawa Y, Murakami M, Tohyama M, Shirakata Y, et al. Eccrine sweat contains IL-1 $\alpha$, IL-1 $\beta$ and IL-31 and activates epidermal keratinocytes as a danger signal. PLoS One. 2013 Jul;8(7):e67666.

29 Marques-Deak A, Cizza G, Eskandari F, Torvik S, Christie IC, Sternberg EM, et al.; Premenopausal, Osteoporosis Women, Alendronate, Depression Study Group. Measurement of cytokines in sweat patches and plasma in healthy women: validation in a controlled study. J Immunol Methods. 2006 Aug;315(1-2):99-109.

30 Nestle FO, Di Meglio P, Qin JZ, Nickoloff BJ. Skin immune sentinels in health and disease. Nat Rev Immunol. 2009 Oct; $9(10): 679-91$.

31 Trivedi DK, Sinclair E, Xu Y, Sarkar D, Walton-Doyle C, Liscio C, et al. Discovery of volatile biomarkers of Parkinson's disease from sebum. ACS Cent Sci. 2019 Apr;5(4):599-606.

32 McGrath J, Saha S, Chant D, Welham J. Schizophrenia: a concise overview of incidence, prevalence, and mortality. Epidemiol Rev. 2008;30(1):67-76.

33 McEvoy JP. The importance of early treatment of schizophrenia. Behav Healthc. 2007 Apr;27(4):40-3.

34 Smith K, Sines JO. Demonstration of a peculiar odor in the sweat of schizophrenic patients. AMA Arch Gen Psychiatry. 1960 Feb;2(2):184-8.

35 Raiszadeh MM, Ross MM, Russo PS, Schaepper MA, Zhou W, Deng J, et al. Proteomic analysis of eccrine sweat: implications for the discovery of schizophrenia biomarker proteins. J Proteome Res. 2012 Apr;11(4):212739.

36 Kharroubi AT, Darwish HM. Diabetes mellitus: The epidemic of the century. World J Diabetes. 2015 Jun;6(6): 850-67.

37 Moyer J, Wilson D, Finkelshtein I, Wong B, Potts R. Correlation between sweat glucose and blood glucose in subjects with diabetes. Diabetes Technol Ther. 2012 May;14(5):398-402.

38 Naehrig S, Chao CM, Naehrlich L. Cystic fibrosis. Dtsch Arztebl Int. 2017 Aug;114(33-34):564-74.

39 Rosenstein BJ, Cutting GR; Cystic Fibrosis Foundation Consensus Panel. The diagnosis of cystic fibrosis: a consensus statement. J Pediatr. 1998 Apr;132(4):589-95.

40 Yosipovitch G, Reis J, Tur E, Blau H, Harell D, Morduchowicz G, et al. Sweat electrolytes in patients with advanced renal failure. J Lab Clin Med. 1994 Dec;124(6):808-12.

41 Willis CM, Britton LE, Harris R, Wallace J, Guest CM. Volatile organic compounds as biomarkers of bladder cancer: sensitivity and specificity using trained sniffer dogs. Cancer Biomark. 2010-2011;8(3):145-53.

42 Junqueira H, Quinn TA, Biringer R, Hussein M, Smeriglio C, Barrueto L, et al. Accuracy of canine scent detection of non-small cell lung cancer in blood serum. J Am Osteopath Assoc. 2019 Jun;119(7):413.

43 Calderón-Santiago M, Priego-Capote F, Turck N, Robin X, Jurado-Gámez B, Sanchez JC, et al. Human sweat metabolomics for lung cancer screening. Anal Bioanal Chem. 2015 Jul;407(18):5381-92. 
Brasier and Eckstein: Digital Biomarkers in Sweat

44 Kintz P, Tracqui A, Mangin P, Edel Y. Sweat testing in opioid users with a sweat patch. J Anal Toxicol. 1996 Oct; 20(6):393-7.

45 Pichini S, Navarro M, Pacifici R, Zuccaro P, Ortuño J, Farré M, et al. Usefulness of sweat testing for the detection of MDMA after a single-dose administration. J Anal Toxicol. 2003 Jul-Aug;27(5):294-303.

46 de la Torre R, Pichini S. Usefulness of sweat testing for the detection of cannabis smoke. Clin Chem. 2004 Nov; 50(11):1961-2.

47 Marchei E, Farré M, Pardo R, Garcia-Algar O, Pellegrini M, Pacifici R, et al. Usefulness of sweat testing for the detection of methylphenidate after fast- and extended-release drug administration: a pilot study. Ther Drug Monit. 2010 Aug;32(4):508-11.

48 Høiby N, Jarløv JO, Kemp M, Tvede M, Bangsborg JM, Kjerulf A, et al. Excretion of ciprofloxacin in sweat and multiresistant Staphylococcus epidermidis. Lancet. 1997 Jan;349(9046):167-9.

49 Tsunoda M, Hirayama M, Tsuda T, Ohno K. Noninvasive monitoring of plasma L-dopa concentrations using sweat samples in Parkinson's disease. Clin Chim Acta. 2015 Mar;442:52-5.

50 Coravos A, Khozin S, Mandl KD. Developing and adopting safe and effective digital biomarkers to improve patient outcomes. NPJ Digit Med. 2019;2(1):14.

51 Babrak LM, Menetski J, Rebhan M, Nisato G, Zinggeler M, Brasier N, et al. Traditional and digital biomarkers: Two worlds apart? Digit Biomark. 2019;3(2):92-102.

52 Lonini L, Dai A, Shawen N, Simuni T, Poon C, Shimanovich L, et al. Wearable sensors for Parkinson's disease: Which data are worth collecting for training symptom detection models. NPJ Digit Med. 2018;1(1):64.

53 Brasier N, Raichle CJ, Dörr M, Becke A, Nohturfft V, Weber S, et al. Detection of atrial fibrillation with a smartphone camera: first prospective, international, two-centre, clinical validation study (DETECT AF PRO). Europace. 2019 Jan;21(1):41-7.

54 Yun K, Lee J, Choi J, Song IU, Chung YA. Smartphone-based point-of-care lipid blood test performance evaluation compared with a clinical diagnostic laboratory method. arXiv e-prints [Internet]. 2018. Available from: https://ui.adsabs.harvard.edu/abs/2018arXiv180407387Y.

55 Lee JB, Kim JH, Murota H. Perspiration functions in different ethnic, age, and sex populations: Modification of sudomotor function. Curr Probl Dermatol. 2016;51:109-19.

56 Hladek MD, Szanton SL, Cho YE, Lai C, Sacko C, Roberts L, et al. Using sweat to measure cytokines in older adults compared to younger adults: A pilot study. J Immunol Methods. 2018 Mar;454:1-5.

57 Mannini A, Sabatini AM. Machine learning methods for classifying human physical activity from on-body accelerometers. Sensors (Basel). 2010;10(2):1154-75.

58 Chen Y, Lu S, Zhang S, Li Y, Qu Z, Chen Y, et al. Skin-like biosensor system via electrochemical channels for noninvasive blood glucose monitoring. Sci Adv. 2017 Dec;3(12):e1701629.

59 Farrell PM, White TB, Ren CL, Hempstead SE, Accurso F, Derichs N, et al. Diagnosis of cystic fibrosis: Consensus guidelines from the Cystic Fibrosis Foundation. J Pediatr. 2017;181S:S4-S15.e1. 